\title{
HIT Intermediate-Band Solar Cells with Self-Assembled Colloidal Quantum Dots and Metal Nanoparticles
}

\author{
Ignacio Tobías , Manuel J. Mendes `, Alfredo Boronat, Esther López, Pablo García-Linares `, Irene \\ Artacho, Antonio Martí, Santiago Silvestre and Antonio Luque
}

\begin{abstract}
The particular opto-electronic properties of chemically synthesized colloidal nanoparticles can be promising for functional materials, as those required for high efficient photovoltaic (PV) devices. In particular, appropriately-designed semiconductor colloids (quantum dots, QDs) can potentially allow sub-bandgap current generation in intermediate-band solar cells; while metal nanoparticles (MNPs) sustaining surface plasmons can provide both near and far-field light trapping to further boost the generated power. However, the incorporation of colloidal particles in inorganic PV materials is not trivial, therefore their implementation has so far been restricted to organic/polymeric based solar cells. In this work, $\mathrm{PbS}$ colloidal QDs have been incorporated in the intrinsic a-Si:H layer of HIT (substrate/a-Si:H hetero-junction) test structures. Both c-Si and GaAs substrates have been used, and in some cases colloidal Au NPs have also been included. The obtained devices are meant as probes to verify the feasibility of incorporating foreign nanoparticles in a cell structure and not as potentially efficient solar cells. Despite the radical novelties incorporated, the devices behaved similarly to the references, thus proving the compatibility of the proposed materials and processes.
\end{abstract}

Index Terms - Nanoparticles, photovoltaic cells, plasmons, PV quantum dots, nanowires, and quantum wells.

\section{INTRODUCTION}

Intermediate band solar cells (IBSCs) were proposed in 1997 [1] and shown to be theoretically capable of overpassing the so called Shockley-Queisser efficiency limit [2] that applies to conventional mono-junction, two band solar cells. The concept has received considerable attention since then and the operation principles (namely, current generation via two-photon absorption with open circuit voltage greater than the threshold energy) have been proven in a number of devices and material systems: see [3] for a recent review of experimental results on IB devices.

The Stranski-Krastanov (SK) self-assembling method, for epitaxially growing quantum dots (QDs) in III-V semiconductors, was the first IB implementation technique $[4,5]$ whereby nanometer-sized heterostructures are formed inside crystalline layers. The three dimensional confining potentials within such nanostructures support localized energy levels in the otherwise forbidden gap of the host semiconductor that perform as the states of the intermediate band. These localized states can promote generation of photocurrent from photons with energy below the bandgap of the host material, without voltage reduction, thereby enabling a broader use of the solar spectrum and higher cell efficiency. Photovoltaics based on colloidal quantum dots (CQDs) is a growing topic because of the interesting properties of CQDs that hold promise for solar cell applications under many different implementations [6]. Relative to SK self-assembled nanostructures, colloidal dots offer several advantages such as technological control of size and composition; highly monodisperse spherical geometries; tunability of photonic transition energies with dot size; ease of handling in dispersion; low temperature, large area, high throughput processing; wide range of candidate materials, etc. It is then natural to explore the potential of CQDs to fabricate IB solar cells [7].

To produce IB cells, the QDs must be inserted in a semiconductor host with appropriate bandgap. Low processing temperatures are needed so that CQDs are not degraded, and the crystallinity of the host layer must not be an issue since the insertion of foreign nanoparticles would distort it. Sub-bandgap current generation through CQDs in semiconductor polymer hosts for infrared photodetection has been already shown, resembling an IB absorber [8]. Nonetheless, several other choices can be promising for the host material (organic semiconductors, chalcogenide compounds, metal oxides, organo-metal halides, etc).

Amorphous silicon (a-Si:H) is also a good candidate because of its near-optimum bandgap for IBSC operation [1] and the possibility of being processed under $200^{\circ} \mathrm{C}$. In this study, the intrinsic a-Si:H layer in the front contact of a Heterojunction with Intrinsic Thin layer (HIT) solar cell is used as the high bandgap region housing the colloidal QDs that provide the intermediate band levels. HIT solar cells were introduced by Sanyo in 1991 [9] and are today a welldeveloped, high-efficiency industrial technology; used here as test devices to investigate the feasibility of including a planar CQD array, deposited by wet coating methods, in the middle of the cells growth process.

On the other hand, existing IB solar cells based on SK QDs grown epitaxially, have not attained high efficiencies partly due to weak light absorption in the dots [10]. In order to 
remedy this, the electric field enhancement provided by surface plasmon resonances in metal nanoparticles has been invoked, among other techniques $[11,12]$. Noble metals $\mathrm{Au}$ and $\mathrm{Ag}$ ) are best suited to this purpose and nanometer-sized particles are available in colloidal dispersions, which opens the interesting perspective of incorporating both CQDs and metal nanoparticles (MNPs) via wet coating methods $[7,13]$ to fabricate IB devices with plasmon-enhanced absorption.

This paper deals with the fabrication of IB solar cells by inserting arrays of $\mathrm{PbS} C Q D$ s inside amorphous silicon layers in HIT cells. Gold ( $\mathrm{Au})$ MNPs for plasmon enhancement of absorption are also embedded in some devices. The structure and the fabrication procedure are described in Section II. Electrical measurements (IV curve and spectral response) are presented and analyzed in Section III. Conclusions drawn are given in Section IV.
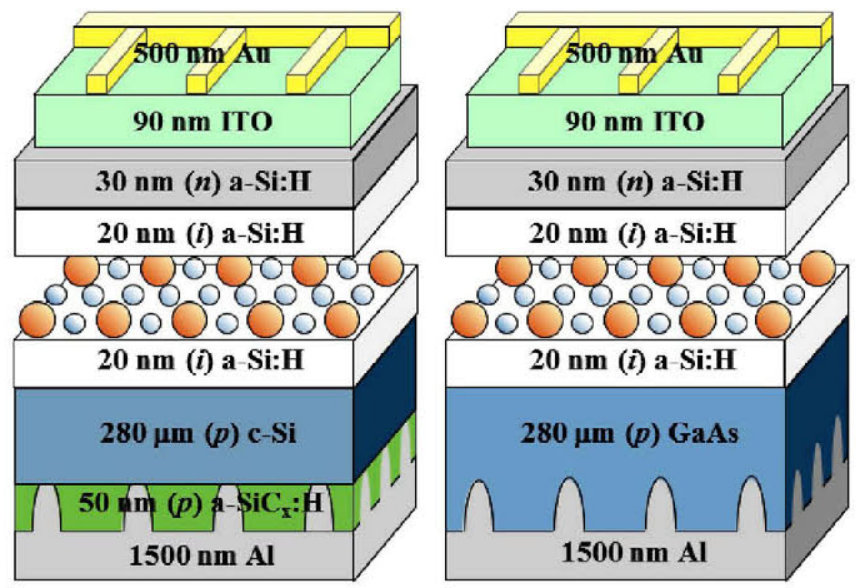

Fig. 1. Fabricated HIT structures on p-Si (left) and p-GaAs (right) crystalline substrates. The smaller blue circles represent quantum dots, and the larger orange circles represent metal nanoparticles.

\section{HIT SOLAR CELLS WITH COLLOIDAL QUANTUM DOTS AND METAL NANOPARTICLES}

\section{A. Description of the Structure}

Heterojunction with Intrinsic Thin layer (HIT) solar cells are based on a crystalline silicon substrate but incorporate amorphous silicon contact layers: it is in these layers that the CQDs are inserted in the work described here, thus constituting the IB material layer, as shown in

Fig. 1 where both CQDs and MNPs are sketched. The thickness of the intrinsic a-Si:H layer is much higher than that (usually a few nanometers) of conventional HIT devices in order to host the nanoparticles. This test structure is used as a probe, to verify the possibility of producing working PV devices with incorporated colloids of different types, since it is produced at low temperatures and it is relatively well known and characterized.

For efficient IB operation the energy band diagram of the CQD/a-Si:H material should resemble that depicted in Fig. 2, where the IB levels are formed by the 1st confined state of the
QDs conduction band. Ideally, all other conduction band states should merge with the host conduction band, and the 1st hole state should line up with the host valence band edge. The symmetric interchanging of the valence and conduction band roles is also acceptable [1]. Hence, the fundamental radiative transition of the quantum dot constitutes one of the steps of the two-photon generation process in IB operation. However, the real band alignment obtained in the CQD/a-Si:H material has not been investigated.

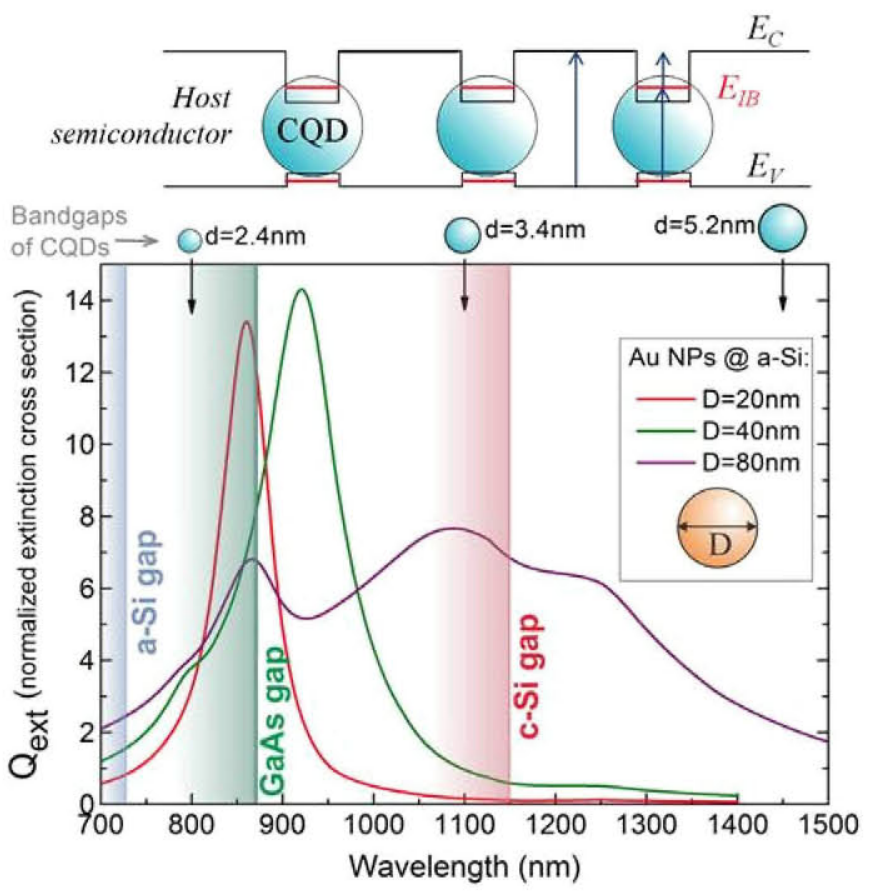

Fig. 2. Top: Schematic band diagram of an IB material constituted by colloidal quantum dots in a host semiconductor, with the intermediate band energy EIB corresponding to the first confined electron state in the dots. Vertical arrows denote photon-induced transitions. Bottom: Normalized extinction (scattering plus absorption) cross section of gold nanospheres embedded in an a-Si medium, calculated with Mie theory. The bandgaps of the CQDs, host (a-Si) and substrate ( $\mathrm{Si}, \mathrm{GaAs})$ materials are also indicated.

The CQDs used are $\mathrm{PbS}$ with 2.4, 3.4 and $5.2 \mathrm{~nm}$ diameters (d). In dispersion, these three types of CQDs exhibit the fundamental transition energies indicated in Fig. 2. These energies are sensitive to the environment and hence somewhat different in the silicon host. In the same figure, the spectra of the extinction (absorption plus scattering) cross section of $\mathrm{Au}$ NPs of different diameters $(D)$, normalized by the particles' area, is plotted $\left(Q_{E X T}\right)$. The spectra were calculated with a Mie theory formalism [14] considering isolated $\mathrm{Au}$ nanospheres embedded in an homogeneous a-Si medium.

As can be seen in Fig. 2, the fundamental transition energies of 2.4 and $3.4 \mathrm{~nm}$ diameter QDs fall within the resonance curves of MNPs and so generation could be assisted by plasmon action. However, they are in the absorption range of the $\mathrm{c}-\mathrm{Si}$ substrate and it would be impossible to detect contributions of photocurrent originating at the QDs. On the other hand, for the largest QDs $(d=5.2 \mathrm{~nm})$ the reverse situation is found. For these reasons, GaAs- 
substrate HIT solar cells have also been fabricated, since in this case the absorption by the QDs - except for the smallest ones - could in principle be detected.

\section{B. Fabrication Procedure}

Si-HIT solar cells have been implemented on boron doped, $0.4 \Omega \cdot \mathrm{cm}$ (approx. $410^{16} \mathrm{~cm}^{-3}$ ) p-type Czochralski substrates. $\mathrm{N}$-type substrates are much more common in HIT cells, but ptype has been used due to the availability of an effective Albased contact system that uses laser to make point contacts to the base through the $\mathrm{a}-\mathrm{SiC}_{\mathrm{x}}: \mathrm{H}$ rear layer. The fabrication sequence is schematized in Fig. 3. A second batch of cells was fabricated on GaAs substrates which are transparent for the fundamental transitions of the larger $d=5.2 \mathrm{~nm}$ CQDs, as indicated in Fig. 2. The a-Si/c-GaAs heterojunction cells are fabricated as described in Fig. 3, sharing most steps with $\mathrm{Si}$ cells except the back layers. Laser is again employed to guarantee ohmic rear contact at low temperature. In fact, the temperature is kept below $200^{\circ} \mathrm{C}$ through the entire processing sequence [15].

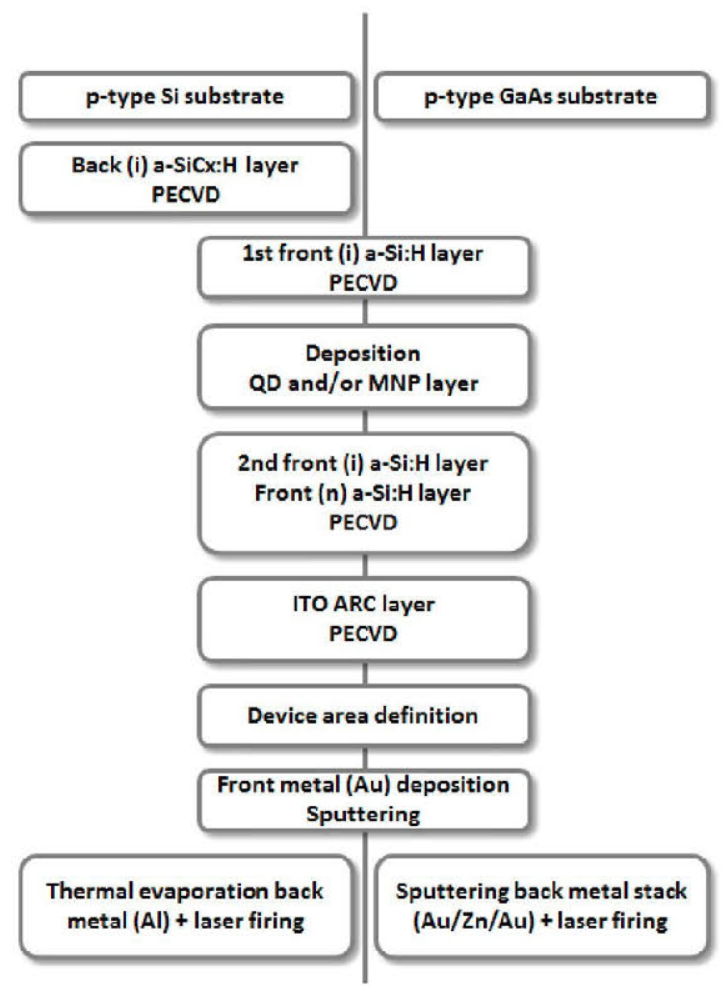

Fig. 3. Processing scheme for HIT cells; on Si substrates on the left, on $\mathrm{GaAs}$ substrates on the right and common steps in the middle.

After the deposition by PECVD of the first 20nm-thick amorphous layer, the samples are taken out of the vacuum chamber and the nanoparticles are deposited with wet-coating procedures described elsewhere [7,12]. Monolayer arrays of evenly-spaced particles on the a-Si surface are obtained. When CQDs and MNPs are both deposited, the arrays are regularly interpenetrated. The distance between the colloidal particles is a few nanometers as determined by the electrostatic repulsion caused by their negatively-charged stabilizing organic capping agents (oleic acid molecules for the CQDs and citric acid for the MNPs). The organic compounds are then burnt-off and the samples loaded again in the CVD reactor to resume the structure formation.

Devices with $25 \mathrm{~mm}^{2}$ total area have been fabricated with different combinations of $\mathrm{Au}$ MNPs and PbS CQDs, as described in TABLE I. Two kinds of reference cells without particles were processed: the REFmod cells, that were extracted from the chamber after the $1^{\text {st }}$ front a-Si layer deposition (see Fig. 3) and passed by the same wet-coating processes of the samples patterned with colloids; and REF cells, also extracted from the chamber at the same time but not exposed to the wet processes.

\section{RESULTS AND DISCUSSION}

IV curves were recorded under xenon lamp illumination approaching standard conditions. Fig. 4 shows the measured IV curves on Si substrates with a striking $\mathrm{S}$ shape. All cell types show a similar behavior, including the references, so such feature is not related to the presence of nanoparticles. Given this shape, the short-circuit current is not indicative of carrier generation and collection processes, and for this reason in TABLE I the saturated photocurrent under reverse bias, $J_{L}$, is given along with the open circuit voltage $V_{O C}$.

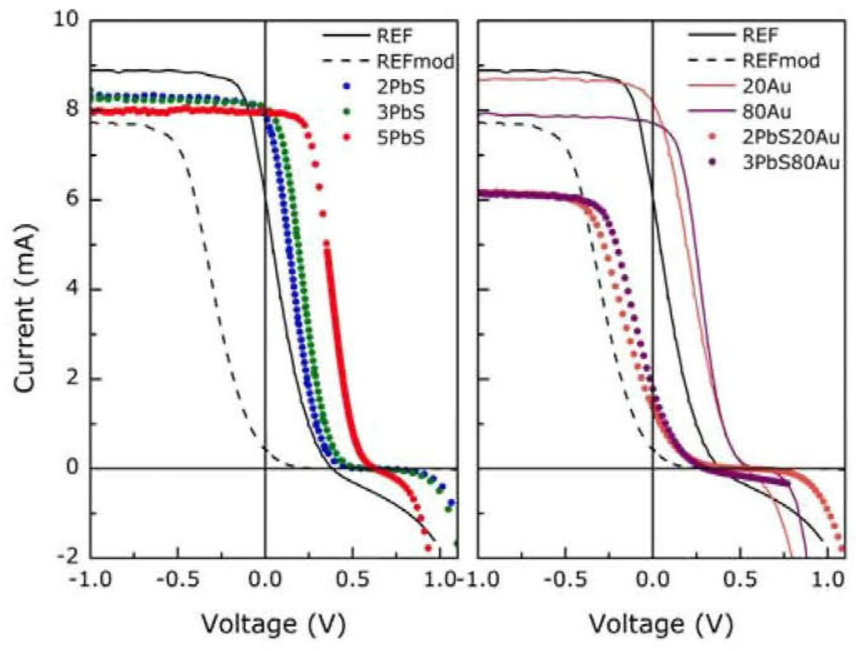

Fig. 4. IV curves of HIT cells on Si substrates with CQDs (left) and with MNPs and MNPs+CQDs (right).

The $\mathrm{S}$ shape reveals a rectifying junction in series, but opposing the cell junction. There are a number of possible causes for it $[16,17]$, including barriers at metallic contacts or interface effects that can appear if the samples are taken out of the vacuum chamber during fabrication. Since the S-shape is in our case common to reference cells, and to silicon and GaAs substrate cells, it seems that the ITO/a-Si:H interface band line-up can be the cause of this behavior.

The photogenerated currents appear to be smaller in the samples with both QDs and MNPs and similar in the remaining categories. Even though the $J_{L}$ of all the cells with QDs or MNPs is lower than that of the REF cells, it is higher 
than the $J_{L}$ of the REFmod cells which passed by the wetcoating processes.
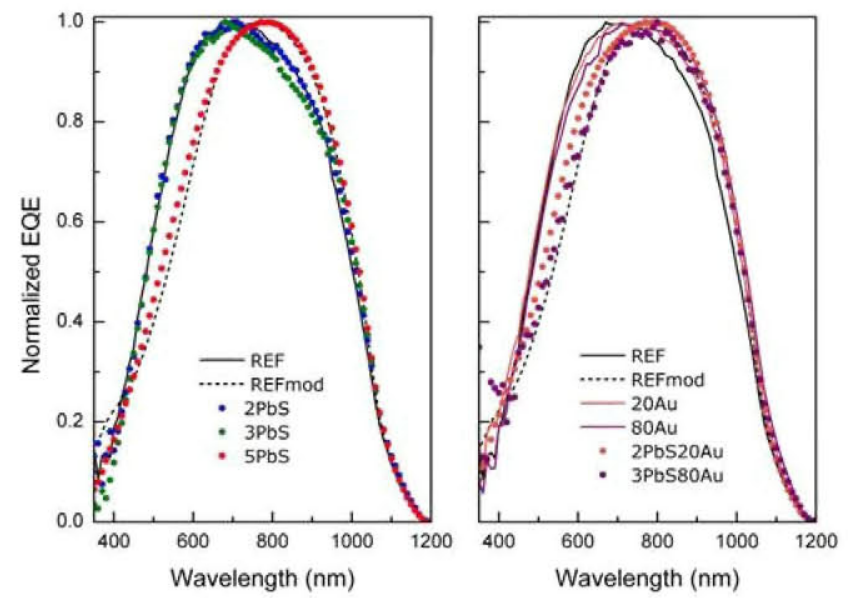

Fig. 5. Normalized external quantum efficiency of cells on $\mathrm{Si}$ substrates. Left: HIT cells with CQDs. Right: cells with MNPs and MNPs+CQDs.

An insight into the current generation behavior of the cells can be gained by examining the (normalized) external quantum efficiencies (EQE), measured on the saturated portion of the curves at a reverse bias of $-1.5 \mathrm{~V}$, given in Fig. 5. There is no detectable sub-bandgap contribution to the photocurrent, which in the case of Si substrates could only, in principle, be detected for the largest $5 \mathrm{~nm}$ QDs absorption.

In practical IB cells this contribution to the $E Q E$ is measurable due to thermal assistance in completing the transition from the IB to the host conduction band $[5,18]$. Since the EQE measurements are performed via monochromatic illumination, the absence of sub-bandgap current generation in the measurements does not mean that the QDs are not behaving as desired. It is possible that their fundamental transition level is located deep inside the $\mathrm{a}-\mathrm{Si}$ bandgap, thus creating a high energy gap $\left(E_{I B}-E_{C}\right.$ as shown in Fig. 2) that impedes the completion of the transition by thermal escape. Another more likely reason is that the light absorption produced by only one layer of nanoparticles in the structure is too low to be detected.

On the other hand, in the useful spectrum region the differences in quantum efficiency are not related to cell structure (references, quantum dot- and metal nanoparticlecontaining cells). In particular, light scattering at metal nanoparticles is known to improve infrared absorption in the far field by tilting the ray paths inside the substrate $[19,13]$. The largest $\mathrm{Au}$ nanoparticles used here $(D=80 \mathrm{~nm})$ are, in principle, appropriate to scatter light in the $1000-1100 \mathrm{~nm}$ range, as shown in Fig. 2. However, no enhancement can be observed for these samples in the EQE measurements.

Devices made on GaAs substrates show also photovoltaic behavior, as can be appreciated in the IV curves shown in Fig. 6. The S-shape is again found, but in this case the current fails to saturate at a given reverse threshold, showing leaky behavior and even breakdown in some samples.
Photocurrents in TABLE I are determined by linear extrapolation of the quasi-saturated reverse portion of the IV curve. Samples containing CQDs present higher currents than references, and while the devices with MNPs appear to be in reverse breakdown before saturation is reached, the rectifying action of the cell junction seems to be restored for those containing CQDs and MNPs.

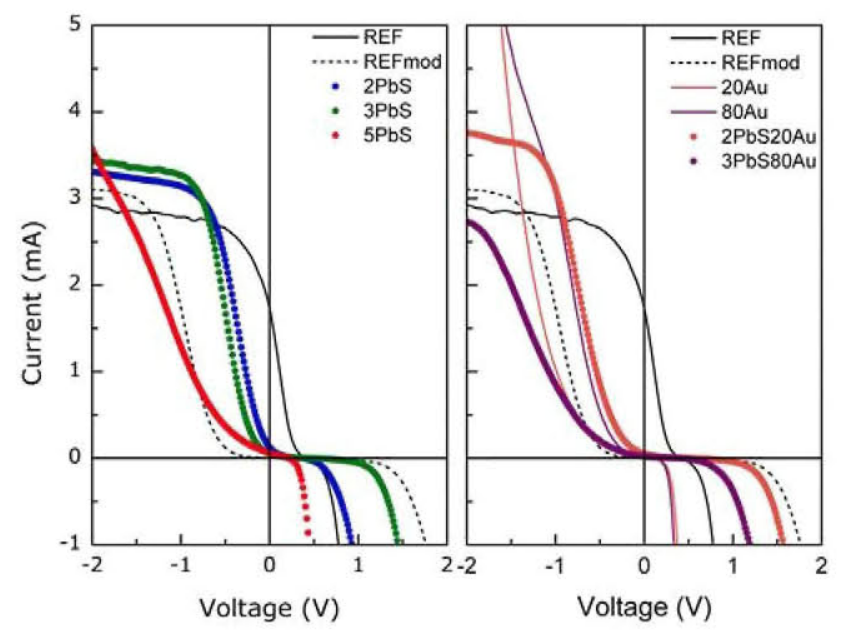

Fig. 6. IV curves of HIT cells on GaAs substrates.
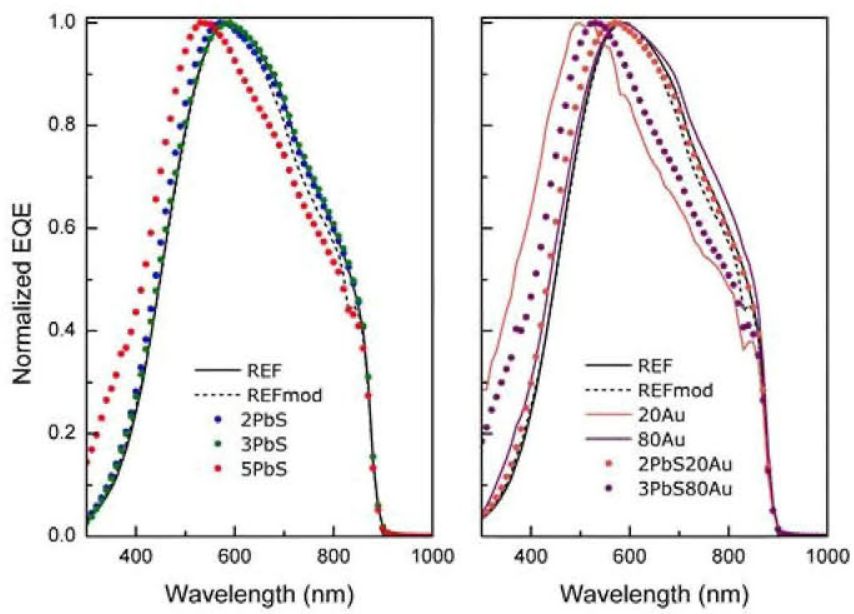

Fig. 7. Normalized external quantum efficiency of HIT cells on GaAs substrates.

The normalized external quantum efficiencies under reverse bias are shown in Fig. 7, revealing again no appreciable current generation through the quantum dot fundamental transition and similar behavior, with apparently no distinct features related to structure, in the useful spectral region.

\section{CONCLUSIONS}

Photovoltaic test devices based on the HIT structure have been fabricated that incorporate $\mathrm{PbS}$ colloidal quantum dots in the intrinsic a-Si:H region of the front contact and/or $\mathrm{Au}$ colloidal nanoparticles. The devices are not meant as potentially efficient solar cells but rather as a first step to test 
the feasibility of incorporating these foreign particles in the cells material, in order to potentially exploit their particular physical properties respectively for intermediate band and plasmonic light trapping effects.

The most remarkable result is that, despite the radical novelties of the structure, all samples behaved as solar cells with both $\mathrm{Si}$ and GaAs subtrates, in a similar way to the reference devices. This shows the compatibility of the materials and processes proposed here. In this respect, note that the size of the metal nanoparticles is similar or even larger than the thickness of the a-Si:H region where they are embedded, and despite this and the corresponding deformation the samples performance is not much affected.

In all cases the IV curves present marked S-shapes so that fill factors and efficiencies are very low; however, this is not due to the presence of nanoparticles as it affects reference cells too. In fact, Au nanoparticles have been reported to cure $\mathrm{S}$-shape IV curves in a-SiC:H solar cells of similar structures through work-function adjustment [20].

Nanoparticles degrade neither the open circuit voltage nor the current collection, as seen in the external quantum efficiencies, so that it seems that large amounts of defects, whether at the interfaces or in the volume, have not been introduced.

On the other hand, the contribution of photo-induced transitions through the quantum dot levels to the cell current cannot be detected. This is attributable to the very low absorption that a single layer of nanoparticles can provide.

\section{REFERENCES}

[1] A. Luque and A. Martí, "Increasing the Efficiency of Ideal Solar Cells by Photon Induced Transitions at Intermediate Levels". Physical Review Letters 78 (1997) 5014-5017.

[2] W. Shockley and H.J. Queisser, "Detailed Balance Limit of Efficiency of p-n Junction Solar Cells". Journal of Applied Physics 32 (1961) 510-519.

[3] I. Ramiro, A. Martí, E. Antolín and A. Luque, "Review of Experimental Results Related to the Operation of Intermediate Band Solar Cells". IEEE Joumal of Photovoltaics 4 (2014) 736-748.

[4] A. Martí, L. Cuadra and A. Luque, "Quantum dot intermediate band solar cell", Proc. of the 28th IEEE Photovoltaic Specialists Conference (2000) 940-943.

[5] A. Luque, A. Martí, C. Stanley, N. López, L. Cuadra, D. Zhou, J. L. Pearson and A. McKee, "General equivalent circuit for intermediate band devices: potentials, currents and electroluminescence". Journal of Applied Physics 96 (2004) 903-909.

[6] I. J. Kramer and E. H. Sargent, "The Architecture of Colloidal Quantum Dot Solar Cells: Materials to Devices". Chemical Reviews 114 (1) (2014) 863-882.

[7] M. J. Mendes, E. Hernández, E. López, P. García-Linares, I. Ramiro, I. Artacho, E. Antolín, I. Tobías, A. Martí and A. Luque, "Self-organized colloidal quantum dots and metal nanoparticles for plasmon-enhanced intermediate-band solar cells". Nanotechnology 24 (34) (2013) 345402.

[8] A. Stiff-Roberts, D. Adrienne and K. Lantz, "Roomtemperature, intraband, infrared absorption in CdSeppoly [2methoxy -5- (2'-ethylhexyloxy) -1,4- (1-cyanovinylene)
For this concept to become a feasible route to intermediate band solar cells, the technology must be developed to include many quantum dot - metal nanoparticle layers.

TABLE I. HIT SOLAR CELLS WITH CQDS AND/OR MNPS IN THE A-SI:H LAYER.

\begin{tabular}{|c|c|c|c|c|c|}
\hline Sample name & Substrate & $\begin{array}{l}\text { PbS QDs } \\
\text { diameter }\end{array}$ & $\begin{array}{c}\text { Au } \\
\text { MNPs } \\
\text { diameter }\end{array}$ & $\begin{array}{c}I_{L} \\
(\mathbf{m A})\end{array}$ & $\begin{array}{c}V_{O C} \\
(\mathrm{mV})\end{array}$ \\
\hline SiREF & $\mathrm{Si}$ & - & - & 8.9 & 369 \\
\hline SiREFmod & $\mathrm{Si}$ & - & - & 7.7 & 518 \\
\hline $\mathrm{Si2PbS}$ & $\mathrm{Si}$ & $2.1 \mathrm{~nm}$ & - & 8.3 & 612 \\
\hline $\mathrm{Si3PbS}$ & $\mathrm{Si}$ & $3.4 \mathrm{~nm}$ & - & 8.4 & 603 \\
\hline $\mathrm{Si} 5 \mathrm{PbS}$ & $\mathrm{Si}$ & $5.1 \mathrm{~nm}$ & - & 8.0 & 629 \\
\hline $\mathrm{Si} 20 \mathrm{Au}$ & $\mathrm{Si}$ & - & $20 \mathrm{~nm}$ & 8.7 & 531 \\
\hline $\mathrm{Si80Au}$ & $\mathrm{Si}$ & - & $80 \mathrm{~nm}$ & 7.9 & 592 \\
\hline $\mathrm{Si} 2 \mathrm{PbS} 20 \mathrm{Au}$ & $\mathrm{Si}$ & $2.1 \mathrm{~nm}$ & $20 \mathrm{~nm}$ & 6.2 & 551 \\
\hline $\mathrm{Si} 3 \mathrm{PbS} 80 \mathrm{Au}$ & $\mathrm{Si}$ & $3.4 \mathrm{~nm}$ & $80 \mathrm{~nm}$ & 6.1 & 300 \\
\hline GaAsREF & GaAs & - & - & 2.6 & 417 \\
\hline GaAsREFmod & GaAs & - & - & 2.9 & 273 \\
\hline $\mathrm{GaAs} 2 \mathrm{PbS}$ & $\mathrm{GaAs}$ & $2.1 \mathrm{~nm}$ & - & 3.0 & 356 \\
\hline GaAs3PbS & GaAs & $3.4 \mathrm{~nm}$ & - & 3.1 & 423 \\
\hline $\mathrm{GaAs} 5 \mathrm{PbS}$ & $\mathrm{GaAs}$ & $5.1 \mathrm{~nm}$ & - & - & 210 \\
\hline $\mathrm{GaAs} 20 \mathrm{Au}$ & $\mathrm{GaAs}$ & - & $20 \mathrm{~nm}$ & - & 124 \\
\hline $\mathrm{GaAs} 80 \mathrm{Au}$ & GaAs & - & $80 \mathrm{~nm}$ & - & 68 \\
\hline $\mathrm{GaAs} 2 \mathrm{PbS} 20 \mathrm{Au}$ & GaAs & $2.1 \mathrm{~nm}$ & $20 \mathrm{~nm}$ & 3.4 & 501 \\
\hline GaAs3PbS80Au & GaAs & $3.4 \mathrm{~nm}$ & $80 \mathrm{~nm}$ & 2.5 & 370 \\
\hline
\end{tabular}

\section{ACKNOWLEDGEMENTS}

This work has been supported by the Spanish Science Ministry through contract PROMESA ENE2012-37804-C01.

phenylene] nanocomposites drop cast on GaAs". Joumal of Applied Physics 103 (2008) 104316 1-8

[9] K. Wakisaka, M. Taguchi, T. Sawada, M. Tanaka, T. Matsuyama, T. Matsuoka, S. Tsuda, S. Nakano, Y. Kishi and T. Kuwano, "More than $16 \%$ solar cells with a new HIT (doped a$\mathrm{Si} /$ non doped a-Si/c-Si) structure". Proc. of the 22 nd IEEE Photovoltaic Specialists Conference (1991) 887-892.

[10] A. Mellor, A. Luque, I. Tobías and A. Martí, "Realistic detailed balance study of the quantum efficiency of quantum dot solar cells". Advanced Functional Materials 24 (2014) 339-345.

[11] M. J. Mendes, A. Luque, I. Tobías and A. Martí, "Plasmonic light enhancement in the near-field of metallic nanospheroids for application in intermediate band solar cells". Applied Physics Letters 95 (2009) 071105.

[12] M. J. Mendes, E. Hernández, I. Tobias, A. Martí and A. Luque, "Embedment of Metal Nanoparticles in GaAs and $\mathrm{Si}$ for Plasmonic Absorption Enhancement in Intermediate Band Solar Cells". Proc. of the 25th European Photovoltaic Solar Energy Conference (2010) 218-222.

[13] M. J. Mendes, S. Morawiec, F. Simone, F. Priolo and I. Crupi, "Colloidal plasmonic back reflectors for light trapping in solar cells". Nanoscale 6 (2014) 4796-805

[14] Q. Fu and W. Sun "Mie Theory for Light Scattering by a Spherical Particle in an Absorbing Medium." Applied Optics 40 (2001) 1354-61

[15] A. Boronat, S. Silvestre and A. Orpella, "Ohmic contacts fabricated on moderately doped p-type GaAs by sputtering deposition and a laser-firing process," Journal of Vacuum Science \& Technology B: Microelectronics and Nanometer Structures 31 (2013) 051209 1-7

[16] M. Lu, U. Das, S. Bowden, S. Hegedus and R. Birkmire, "Optimization of interdigitated back contact silicon heterojunction solar cells: tailoring hetero-interface band 
structures while maintaining surface passivation". Progress in Photovoltaics 19 (2011) 326-338.

[17] U. Das, S. Hegedus, Z. Lulu, J. Appel, J. Rand and R. Birkmire, "Investigation of hetero-interface and junction properties in silicon heterojunction solar cells". Proc. of the 35th IEEE Photovoltaic Specialists Conference (2010) 1358-1362.

[18] E. Antolín, A. Martí, C.D. Farmer, P. García-Linares, E. Hernández, A. M. Sánchez, T. Ben, S. I. Molina, C. R. Stanley and A. Luque, "Reducing carrier escape in the InAs/GaAs quantum dot intermediate band solar cell". Journal of Applied Physics 108 (2010) 064513-064517.

[19] S. Pillai, K. R. Catchpole, T. Trupke and M. A. Green, "Surface plasmon enhanced silicon solar cells". Journal of Applied Physics 101 (2007) 093105 1-8.

[20] J. Kim, A. Abou-Kandil, K. Fogel, H. Hovel and D. K. Sadana, "The Role of High Work-Function Metallic Nanodots on the Performance of a-Si:H Solar Cells: Offering Ohmic Contact to Light Trapping". ACS Nano 4 (2010) 7331-7336. 